\title{
The effects of culture, environment, age, and musical training on choices of visual metaphors for sound
}

\author{
ROBERT WALKER \\ Simon Fraser University, Burnaby, British Columbia, Canada
}

\begin{abstract}
Studies involving acts of auditory perception are usually concerned with scaling or categorization of stimuli. Cross-modal studies, consequently, involve matching estimations of stimulus magnitude or category across modalities. The present study was concerned with auditory stimulus differentiation among four acoustic parameters (frequency, waveform, amplitude, and duration) and selection of visual metaphors for each. Previous studies had indicated the following crossmodal matchings: frequency with vertical placement, waveform with pattern, amplitude with size, and duration with horizontal length. A total of 838 subjects of varying ages and cultural, environmental, and musical backgrounds were tested by means of an instrument developed from previous studies of this type. No type of visual metaphor other than those listed was significantly supported in the subjects' responses. Consistency of choice was determined predominantly by musical training. Age was also a factor, although to a lesser extent, and cultural and environmental effects were observed in subjects with less exposure to Western life-styles.
\end{abstract}

Studies in auditory perception are typically concerned with pitch or intensity perception, either involving matching with some given standard or requiring various acts of auditory scaling or categorization (e.g., Burns \& Ward, 1974; Harris \& Siegel, 1975). One procedure is to provide a given standard against which subjects are asked to compare different stimuli. The standard is given a numerical value (e.g., 100) and experimental stimuli are judged quantitatively in relation to it and then assigned values correspondingly. Investigations into other perceptual modes use similar techniques, and are concerned with similar estimations of a quantitative nature. Such tasks are intramodal and do not attempt to deal with qualitative matters such as how pitch perception might differ from, say, intensity perception, by virtue of a difference in relationships between acoustical event and type of information extracted. For example, pitch movement is thought of, musically, as something on a vertical, high-low continuum. But this is a subjective interpretation of something that acoustically occurs on a more-less continuum: different repetition rates of a waveform. The concept of intensity, however, seems more related to its acoustical correlate in the sense that acoustical energy and intensity are both concerned with representations of more and less of something on a quantitative scale of values.

Many studies of cross-modal transfer of information are also concerned with estimations of stimulus magnitude or

Field travel and development of materials used in this research were funded by Grant 410-85-0214 from the Social Sciences and Humanities Research Council of Canada. The kind cooperation of schools, Indian Band Councils, the Government of the Northwest Territories, and other authorities and individuals is gratefully acknowledged. The author's address is Faculty of Education, Simon Fraser University, Burnaby, BC V5A 1S6, Canada. category (e.g., Ward, 1985, concerning light to sound). Two such studies (Lewkowicz \& Turkewitz, 1980, 1981) demonstrated that infants were able to match sound intensities with light intensities. These studies showed how the infants' cardiac responses reflected a perception that certain light intensities matched certain auditory ones. In fact, there is ample evidence of innate ability in infants for cross-modal transfer of information (e.g., Bryant, Jones, Claxton, \& Perkins, 1972; Lawson, Ruff, McCartonDaum, Kurtzberg, \& Vaughan, 1984; McGuire \& Turkewitz, 1978; Mendelson \& Ferland, 1982; Rose, Gottfried, \& Bridger, 1983; Walker-Andrews \& Lennon, 1985). This kind of transfer is described as amodal (Lewkowicz \& Turkewitz, 1980), in that a quality such as intensity can be judged in a variety of modalities. It is argued that modal-specific qualities, such as pitch in sound, do not appear to elicit equivalences across modalities in the way that intensity changes in both light and sound are able to, in which case terms such as cross-modal integration or intersensory coordination (Lewkowicz \& Turkewitz, 1980) might be more appropriate.

This paper is concerned with auditory movements in frequency, waveform, amplitude, and duration, and with choices of visual metaphors for these events. Auditory movements in frequency might be regarded as modalspecific in that information from such movements has no simple intermodal application. As already explained, we generally do not think of more or less frequency, yet repetition rates are measured in those terms. One study (Walker, 1987) has shown that it is by no means automatic for some subjects (nonmusicians) to think of frequencies as higher or lower in the visual sense. Waveforms involving more or less complexity of spectral components might be regarded as amodal in the sense that 
they can be replicated visually as more or less complexity of pattern or texture. Movements in amplitude and duration might also be regarded as amodal in that the former is perceived as more or less loud and the latter as longer or shorter, and these perceptions can be replicated visually as more or less and longer and shorter.

This paper is concerned, therefore, with both modalspecific and amodal qualities, and how such qualities are reflected in another modality, specifically between visual and auditory modes of perception. The work reported describes subjects' qualitative judgments in cross-modal transfer and integration of information between auditory and visual modalities. The experimental task was not concerned with stimulus magnitude or category estimation. Instead, subjects were asked to select a visual metaphor for a perceived auditory quality. Specifically, they were asked to differentiate between the four acoustic parameters of frequency, waveform, duration, and amplitude, and to indicate this cross-modally through appropriate choices of visual metaphor (i.e., visual deployments in size, shape, pattern, vertical or horizontal placement, etc.). Previous studies (see below) led one to expect that subjects would choose different visual metaphors for different acoustic parameters and there would be some consistent visually expressed identity for each, but that variables such as subjects' musical training, age, culture, and environment might exercise some effects.

In order to distinguish among different acoustic parameters for experimental purposes, there was a need for some auditory change in each stimulus, but only for parameter identification. For example, two discrete frequencies or amplitudes were used as examples of the auditory classes frequency and amplitude, not as vehicles for estimating the degree of difference within each. Consequently, all alternative choices of visual metaphor for this stimulus reflected the two events quantitatively in this respect.

\section{Previous Investigations of Visual Metaphors for Sounds}

Earlier studies (Walker, 1978, 1981) showed that subjects produced some systematic visual matchings for auditory events. These studies began with investigations of children's invented visual representations for sounds, using as models some new types of musical notations for the music of avant-garde composers in both North America and Europe. Provided one could isolate individual parameters of sound sufficiently, there was some evidence of systematic use of visual metaphor for each auditory parameter in the children's visual creations. The more complex the sound, the more diffuse and arbitrary tended to be the visual representation devised by many groups of children. In one study (Walker, 1978), groups of children were asked to invent their own visual shapes that "looked like" the sounds. Sounds were obtained first from various musical instruments presented live, then from carefully controlled electronically generated sounds.

Further investigation (Walker, 1978, 1981, 1985, 1987) yielded some firm evidence for the following systematic matchings of two-dimensional visual metaphors with acoustic parameters: frequency change was matched with placement along a vertical axis, different waveforms with different visual patterns, different durations with matching horizontal lengths, and amplitude differences with sizes of visual shape.

An investigation using congenitally blind subjects (Walker, 1985) indicated similar proclivities among this population. Statistically, no difference was discovered between the response choices of a large sample of sighted subjects and those of the small sample of congenitally blind subjects tested. The task involved cross-modal matching of auditory stimuli with tactile responses selected from a given set of alternatives. The tactile response choices were three-dimensional versions of those used with the sighted subjects. This finding might be considered evidence for a central processing mechanism that is relatively independent of type of sensory experience (Hall, 1981).

The proclivity for consistently matching specific visual metaphors with specific auditory events, as described above, was confirmed in both free-choice and closedresponse tasks in earlier studies. However, consistency and reliability of response was not always uniform or complete. One study (Walker, 1987) indicated that musical training was a major factor in the choice of a vertically positioned visual metaphor for frequency change, and that this factor was more significant than cultural or ethnic background. Musically naive subjects tended to choose randomly. Some effects of age and intelligence (determined by culture-fair tests) were found initially (Walker, 1978), but subsequent work indicated that the latter factor was not as important as others. In general, there appeared to be less consistency in results from some tasks involving frequency change, and some tasks involving specific changes of amplitude and waveform, than from others. However, since the main purpose of this study was to investigate the previously reported consistency of choice of visual metaphors for sound among varied groups of subjects, it was not intended to investigate extensively the effects of different acoustical configurations within each parameter of sound. It was felt that such matters would demand separate treatment following the more detailed information expected from the present study concerning the effects of different backgrounds of subjects on such cross-modal choices. Accordingly, acoustic stimuli were prepared with the main intention of eliminating as much ambiguity in the auditory event as possible in order to delineate clearly each acoustic parameter. The visual response choices were varied only within the range of alternatives indicated in previous studies.

\section{Previous Response Differences and Commonalities-Innate or Learned?}

It seems clear from a number of investigations that humans, and indeed all animals, have an innate mechanism for processing, storing, and transferring across modalities information relating to auditory movements in am- 
plitude, duration, frequency, and waveform, and various combinations of these. Clarkson and Clifton (1985) showed that 7-8-month-old infants were able to classify tonal complexes into rudimentary pitch categories. Eimas, Siqueland, Jusczyk, and Vigorito (1971) reported infant abilities for categorization of speech phonemes. Olsho, Schoon, Sakai, Turpin, and Sperduto (1982) reported auditory frequency discrimination abilities in infants, as did Wormith, Pankhurst, and Moffitt (1975). But we can say that at such an early age mental schemata resulting from experience are relatively undeveloped, and such acts are due more to the child's innate neural abilities for processing such events than to exhibition of learned response mechanisms. Additionally, many investigators have demonstrated similar abilities in various animals: Halse, Cynx, and Humpal (1984) show them in birds, Javel (1980) in rabbits, and Chung and Colavita (1976) in cats. These findings seem to point to a capacity in innate neural wiring for such discriminations, rather than to some ability acquired through experience. Experience, however, influences the development of such innate abilities.

Terhardt (1974) argued that there are learned effects of speech and language in the shape of auditory gestaits which exert influence in the central processing of sound, particularly in the case of pitch perception. Divenyi (1979), in support of Terhardt, added that there is evidence of such learned effects from brain-damaged children and that environmental sounds, including music, play a part. He pointed out that hearing loss occurring before the formation of such gestalts results in lack of control over vocal pitch and speech, and an inability to acquire any. In the case of music, Divenyi cited the complex inharmonic frequency ratios found in the sound spectra of the Javanese gamelan and the correspondingly complex frequency ratios found in Javanese melodies, as compared with the use of simple harmonic ratios found in Western music.

This would seem to indicate that subjects acculturated in different auditory environments and language traditions might be expected to have formed different auditory gestalts at the higher levels of neural processing. Some signs of these differences might therefore be expected to show in tests involving selections of visual metaphors for different sounds. It might be expected, for example, that some amodal tasks would not require the intervention of higher cognitive processes that are formed by learning and experience. Modal-specific tasks, on the other hand, may require the intervention of such processes. It can also be postulated that all speech and environmental noises contain certain common elements that result in some common gestalt formations. These might also be responsible for some commonalities in the use of visual metaphors for sounds. Some clear indications were seen in one study (Walker, 1987) that certain types of experience (e.g., musical training) are more important influences than others (e.g., language and environment) in auditory/visual in- tegration tasks involving frequency change and vertical visual deployment.

Distinguishing between responses that utilize innate neural wiring and those resulting from higher neural processing, whether from general experience or specific skill training, is indeed a formidable task. This study cannot pretend to address such issues. It is assumed that cognition of either speech or musical sounds will, by its very nature as a product of experience, involve higher or more central processing than will, for example, discrimination of a simple change in frequency. But whether it is possible to ignore experience completely as a factor in the latter activity seems doubtful. In Western music there is a well-established visual metaphor for musical pitch movement in the vertical placement of musical notes on the stave. Obviously, training is a factor in the ability to respond appropriately to such a system of notation. Speech sounds have no such commonly used visual metaphor: written language has no similar type of connection with the auditory nature of speech sounds. A written word is not meant to look like the sound of the spoken word. This fact alone indicates that the effect of learned responses in relation to speech is likely to be different from the effect of learned responses in relation to music. Yet speech comprises complex sounds, as does music, and one speaks of high-pitched or low-pitched voices in common discourse. An interesting question is whether there might be any differential effects of acculturation among different speech cultures, in view of the fact that some languages utilize greater pitch ranges or more frequent pitch variation than do others.

\section{Effects of Musical Training}

A number of studies have shown that trained musicians can be expected to perform differently from musically naive subjects in certain tasks involving pitch magnitude estimation and pitch interval recognition (e.g., Siegel \& Siegel, 1977). There are also a number of similar experimental data relating to amplitude scaling tasks (e.g., Algom \& Marks, 1984; Ward, 1985). One study cited above (Walker, 1987) appears to be unique in that it reports similar effects in cross-modal integration involving frequency and visual vertical placement, which are described above as modal-specific qualities. Another investigator (Ryker, 1946) reported that trained musicians performed less well on pitch judgment tasks involving pure tones than on those involving complex tones obtained from the piano. It seems, therefore, that use of pure tones, at least in tasks involving frequency, would reduce to some degree the advantages musicians might have in this respect. Others have reported that pure tones are processed differently from complex tones (e.g., de Boer, 1976; Roederer, 1979), but that pitch information can be extracted from both pure and complex tones. Few similar reports concerning the other acoustic parameters used in the present study (amplitude, waveform, and duration) 
seem to exist. This may be due to the preeminence of pitch in Western music and a consequent preoccupation with establishing psychoacoustic correlates for pitch perception.

\section{Some Acoustical Considerations}

The association between frequency and pitch is a complex one, but in musical terms frequency change means pitch change. In the literature on acoustics there is firm evidence of the importance of central pitch processing (de Boer, 1976; Houtsma \& Bilsen, 1970; Houtsma \& Goldstein, 1972; Terhardt, 1974) in perceptions of complex tonal stimuli whose fundamentals are different. It is also well established that pitch perception of complex tones is derived from information perceived from at least two adjacent harmonics (Ritsma \& Bilsen, 1970; Roederer, 1979), particularly below the eighth, but position of these crucial harmonics is dependent upon the repetition rate of the fundamental (de Boer, 1976). Since pitch in Western music is derived from complex sounds in this manner, and is represented vertically in musical notation, it was important in the present study that this kind of acoustic information be absent in the frequency class of auditory stimuli. In this way it was intended to avoid disadvantaging musically naive subjects and to eliminate as far as possible the effects of a learned association relating to musical pitch between musical notation and musical sound. The effects of musical experience on perception of other acoustic parameters have not been dealt with to any great extent in the literature.

The choice of waveform was made to eliminate known distortion effects or ambiguities due to interactions of acoustic parameters. Fundamental frequencies of all stimuli were confined to a range of $170-860 \mathrm{~Hz}$, the normal range for voices of children and adults. It was hoped that this would minimize effects of any specialized auditory experience other than normal spoken communication. Simple (or, as they are sometimes known, pure) tones were used predominantly. However, complex tones (i.e., with spectral energy spread over several harmonics in varying strengths) were used in some tests because of known distortion effects of pure tones. For example, changes in amplitude of pure tones give a strong impression of pitch change in certain circumstances.

\section{THE EXPERIMENT}

To investigate the matters raised above, data were needed from subjects with different levels of musical training and with no musical training, with radically different cultural backgrounds and environments, and of widely varied age levels. In addition, a varied set of visual metaphors for sound was needed which would explore further and clarify some issues raised in earlier results. Since earlier studies indicated a proclivity for subjects to match amplitude, waveform, duration, and frequency with visual size, pattern, horizontal length, and vertical placement, respectively, with only these four visual metaphors plus shape occurring for any acoustic parameter, it was possible to eliminate other types of visual movement from consideration. The visual response choices, therefore, comprised four alternatives for each acoustic stimulus: size, pattern, shape, and vertical placement, except in the case of duration, for which horizontal length was substituted for one of the four. The auditory stimuli needed to comprise unambiguous changes in frequency, waveform, duration, and amplitude in order to provide maximum evidence of the nature of the auditory parameter. The auditory experimental variable was therefore confined to the generality of the four acoustic parameters. The detail of variation in each single stimulus item was, accordingly, experimentally less significant than the parameter it signified. Within these constraints 16 sets of auditory stimuli involving four manipulations of four auditory parameters (frequency, amplitude, duration, and waveform) were prepared together with 16 sets of visual responses.

\section{Method}

\section{Subjects}

A total of 838 subjects participated. Five different cultural backgrounds were represented, and a sixth group consisted of urban subjects with musical training. All groups were divided into Age Levels 1,2 , and 3 (elementary school students, high school students, and adults) (see Table 1).

Group 1: Musically trained. Age Level 1 subjects were from three elementary schools in Vancouver, British Columbia, where intensive music programs were available for those children who wanted them. Children were not selected according to special musical abilities; they merely had an interest in music. This was felt to be important in order to minimize the differences between these subjects and children who were musically naive. Age Level 2 subjects were chosen similarly from three high school bands and choirs in Vancouver. Age Level 3 subjects comprised a mixture of graduate musicians, music teachers, and symphony orchestra players. All had had post-secondary training in music.

Group 2: Urban (musically naive). Subjects from Age Levels 1 and 2 were chosen from elementary and high schools in Vancouver whose staffs were willing to participate. Students with any formal training in music were asked to sit out the test. Otherwise, the students were a random mix of abilities and backgrounds found in any typical urban classroom. Age Level 3 comprised a deliberate mixture of backgrounds and ages, ranging from university undergraduates to prison inmates to members of social clubs. Their ages ranged from 21 to 70 years.

Table 1

Subjects by Group and Age Level

\begin{tabular}{l|cccc}
\hline & \multicolumn{3}{c}{ Age Level } & \\
\cline { 2 - 4 } & 1 & 2 & 3 & Total \\
\hline Group 1: Musically Trained & 56 & 49 & 54 & 159 \\
Group 2: Urban & 47 & 47 & 46 & 140 \\
Group 3: Inuit & 44 & 53 & 48 & 145 \\
Group 4: Haida & 52 & 37 & 44 & 133 \\
Group 5: Shuswap & 48 & 35 & 34 & 117 \\
Group 6: Tsimsian & 44 & 38 & 62 & 144 \\
Total & & & & 838 \\
\hline
\end{tabular}


Group 3: Inuit. These subjects were located on Baffin Island in northeastern Canada. Subjects from Age Levels 1 and 2 were all status native Inuit in elementary and secondary schools in Frobisher Bay. It is the practice on Baffin Island to educate all children over Grade 5 in Frobisher Bay; therefore, the sample contained children from all over the island. Subjects from Age Level 3 were status Inuit adults from the Inuit Teachers Program at Frobisher Bay, the Remand Centre, a trade school center, and several locations on Baffin Island. All of these subjects were used to communicating in both Inuktituk and English.

Group 4: Haida Indians. These subjects were status Haida Indians on the Queen Charlotte Islands off the coast of northern British Columbia. All spoke English and the Haida language in varying degrees.

Group 5: Shuswap Indians. These subjects were status Shuswap Indians from communities on two remote reservations (Alkali and Canim) in central British Columbia. A good deal of instruction in schools there is in the Shuswap language, and adults speak Shuswap in daily life. However, all of these subjects spoke English as well.

Group 6: Tsimsian Indians. These subjects were status Tsimsian Indians in remote schools and communities in northwestern British Columbia. The Tsimsian language is used similarly to the Shuswap language; however, the Tsimsian subjects spoke English as well.

\section{Auditory Stimuli}

There were 16 sets of stimuli in all: 4 frequency change, 4 amplitude, 4 duration, and 4 waveform. They were randomized for presentation and numbered 1 to 16 . The sounds were generated electronically using a Roland CS15 synthesizer, and were recorded on magnetic tape using Dolby B sound. The following descriptions of the auditory stimuli are based on analyses derived from software specially designed for use with an IBM microcomputer. This was Micro Speech Lab (University of Victoria, B.C.), which was specially expanded to include chirp $Z$ capability and other types of acoustic analysis.

Frequency. In all cases spectral analysis indicated that over $95 \%$ of total spectral energy lay at the fundamental frequency. The difficulties of producing absolutely pure tones, with $100 \%$ energy at the fundamental, are well documented. The four sets of stimuli were as follows:

1. Two glides ( $571 \mathrm{~Hz}$ to $800 \mathrm{~Hz}$ and reversed) lasting $2 \mathrm{sec}$ each, separated by $1 \mathrm{sec}$ of silence.

2. Two discrete frequencies $(520 \mathrm{~Hz}, 743 \mathrm{~Hz})$ each lasting $1 \mathrm{sec}$, separated by $1 \mathrm{sec}$ of silence.

3. Four discrete sounds $(174 \mathrm{~Hz}, 571 \mathrm{~Hz}, 571 \mathrm{~Hz}, 571 \mathrm{~Hz})$ each lasting $1 \mathrm{sec}$, separated by $1.25 \mathrm{sec}$ of silence.

4. Three glides $(500-580 \mathrm{~Hz}, 580-678 \mathrm{~Hz}$, and $678-852 \mathrm{~Hz}$ ) each lasting $1.5 \mathrm{sec}$, separated by $0.5 \mathrm{sec}$ of silence.

Amplitude. Complex waves were used in all of these stimuli because of known perceptual effects of pitch change when pure waveforms are altered in amplitude. Amplitude was represented as energy in the computer analysis. Energy was calculated as the sum of values within approximately one 25 -msec time frame. The typical jnd for most people with normal hearing is reckoned to be about $2 \mathrm{~dB}$ or $5 \%$. All stimuli contained much greater differences than this. The four sets of stimuli were as follows:

1. Five discrete sounds (all at a fundamental of $656 \mathrm{~Hz}$, and each lasting $1 \mathrm{sec}$ ) separated by $0.4 \mathrm{sec}$ of silence, decreasing in energy by approximately one-third (approximately $7 \mathrm{~dB}$ ) each time, with spectral energy distributed across the first six harmonics in varying amounts as follows: $15 \%$ at harmonic $2,20 \%$ at $3,30 \%$ at 4 , $15 \%$ at 5 , and $20 \%$ at 6 .

2. One continuous sound ( $370 \mathrm{~Hz}$ throughout) increasing, decreasing, and finally increasing in energy (approximate relative values were $28 \mathrm{~dB}, 42 \mathrm{~dB}, 30 \mathrm{~dB}, 45 \mathrm{~dB}$ ) over a 10-sec period, with spectral energy spread as follows: $17 \%$ between harmonics 1 and $2,19 \%$ between 3 and $5,51 \%$ between 6 and 8 , and $9 \%$ between 9 and 10 .

3. Four discrete sounds (all at a fundamental of $274 \mathrm{~Hz}$ ) lasting $1 \mathrm{sec}$, separated by $0.5 \mathrm{sec}$ of silence, with sounds 1 and 3 having five times the energy (approximately $8 \mathrm{~dB}$ difference) of sounds 2 and 4 , and spectral energy spread as follows: $85 \%$ between harmonics 4 and $7,8 \%$ between 9 and 11 , and $5 \%$ between 12 and 15 .

4 . One continuous sound gradually increasing in amplitude eightfold (to approximately $10 \mathrm{~dB}$ ) over $10 \mathrm{sec}$ at a fundamental frequency of $274 \mathrm{~Hz}$, with spectral energy distributed as follows: $5 \%$ at harmonics $4-7,70 \%$ at $8-10$, and $24 \%$ at $11-14$.

Duration. Pure and complex tones were used randomly because there is little known effect on perception of duration from either, provided all other parameters are kept constant. The four sets of stimuli were as follows:

1. Two sounds (at a fundamental of $500 \mathrm{~Hz}$ ) lasting $0.75 \mathrm{sec}$ and $2.4 \mathrm{sec}$, separated by $1.25 \mathrm{sec}$ of silence, with over $95 \%$ of spectral energy at the fundamental.

2. Three sounds (at a fundamental of $160 \mathrm{~Hz}$ ) lasting $1.3,0.5$, and $1.3 \mathrm{sec}$, respectively, separated by $0.5 \mathrm{sec}$ of silence, with over $95 \%$ of spectral energy at the fundamental.

3. Two sounds (at a fundamental of $278 \mathrm{~Hz}$ ) lasting $1 \mathrm{sec}$ and $0.3 \mathrm{sec}$, respectively, separated by $0.5 \mathrm{sec}$ of silence, with spectral energy spread as follows: $51 \%$ at harmonics $1-3,12 \%$ at 5-6, $17 \%$ at $7-9$, and $17 \%$ at $10-13$.

4. Three sounds (at a fundamental of $323 \mathrm{~Hz}$ ) lasting $1.4 \mathrm{sec}$; $3.5 \mathrm{sec}$, and $0.6 \mathrm{sec}$, respectively, separated by $0.5 \mathrm{sec}$ of silence, with over $95 \%$ of spectral energy at the fundamental.

Waveform. Contrast was achieved using complex tones, simple waveforms, and noise in varying combinations. The four sets of stimuli were as follows:

1. Three sounds (same fundamental-260 Hz), each lasting $1 \mathrm{sec}$ and separated by $0.5 \mathrm{sec}$ of silence. Sounds 1 and 3 had over $95 \%$ of spectral energy at the fundamental; sound 2 had spectral energy spread as follows: $7 \%$ at harmonics $1-3,82 \%$ at $4-8$, and $5 \%$ at 9-11.

2. Four sounds (same fundamental $-290 \mathrm{~Hz}$ ), each lasting $1 \mathrm{sec}$ and separated by $0.5 \mathrm{sec}$ of silence. Sounds 1 and 3 were simple waves, with most energy at the fundamental, plus white noise; sounds 2 and 4 were the same simple wave without white noise.

3. Two sounds (same fundamental-328 Hz) each lasting $2 \mathrm{sec}$, and separated by $0.5 \mathrm{sec}$ of silence. Sound 1 was white noise, and sound 2 was a complex wave with a fundamental of $328 \mathrm{~Hz}$ and an energy spread as follows: fundamental, $45 \%$; harmonic $2,12 \%$; $3,9 \% ; 4,10 \%$; and $5,11 \%$.

4. Two sounds (same fundamental-328 Hz), each lasting $1.6 \mathrm{sec}$ and separated by $0.5 \mathrm{sec}$ of silence. Sound 1 was a complex wave with spectral energy spread as follows: $19 \%$ at harmonics $1-3,36 \%$ at $4-6,27 \%$ at $7-9$, and $18 \%$ at $10-12$. Sound 2 had most spectral energy concentrated at the fundamental.

\section{Closed Visual Response Sheets}

For each of the 16 sets of auditory stimuli, the subjects were given a set of four visual metaphors from which to choose. Each set comprised four alternatives: (1) movement along the vertical axis, (2) difference in size, (3) difference in shape, (4) difference in texture or pattern. For duration stimuli, movement along the horizontal axis was substituted for one of the four. Previous studies had indicated that in two-dimensional space, the choice of visual metaphor for these types of auditory events would very likely be contained within such a range of alternatives. The order of the sets of visual metaphors matched the randomized order of the auditory stimuli, and within each set of four the order was randomized throughout. The subjects were asked to identify the selected visual metaphor by drawing a ring around it. Each metaphor choice 

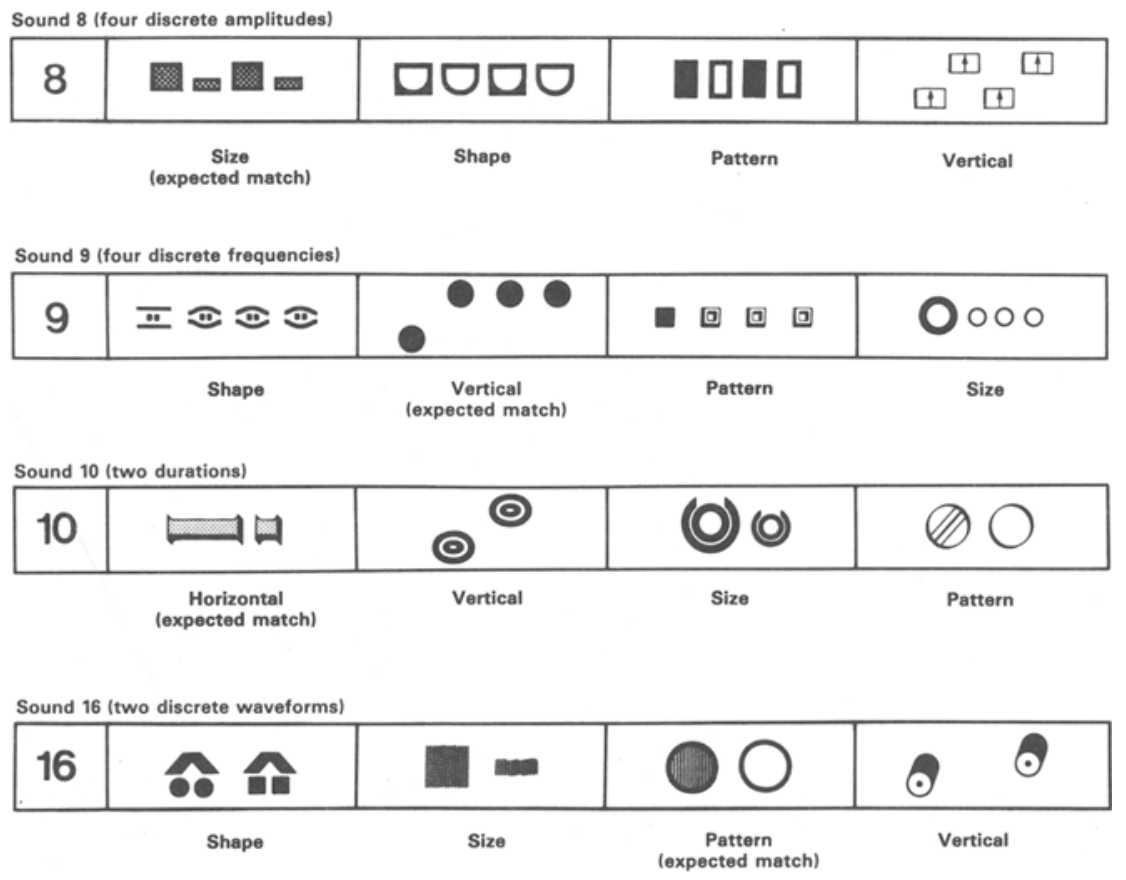

Figure 1. Samples of choices of visual shapes for matching.

matched the stimulus quantitatively; that is, four or two discrete sounds would be matched with alternatives with four or two visual items. Examples of visual response choices are shown in Figure 1 as follows:

Visual metaphors for amplitude. Figure 1, Number 8 (the randomized order position), was presented for matching with Amplitude Stimulus Set 3-four discrete amplitudes (high, SPL, low SPL, high SPL, low SPL).

Visual metaphors for frequency. Figure 1, Number 9, was presented for matching with Frequency Stimulus Set 3-four discrete sounds (one lower in frequency, and three higher yet identical to each other).

Visual metaphors for duration change. Figure 1, Number 10 , was presented for matching with Duration Stimulus Set 3-a long sound followed by a short one.

Visual metaphors for waveforms. Figure 1, Number 16, is presented for matching with Waveform Stimulus Set 4-two discrete waveforms, one extremely complex and the other simple.

Each set of visual response choices in Figure 1 is based on a different graphic design, and this was true for all 16 sets of visual response choices. The question arises, Why employ a different graphic design for each single stimulus item when the same design could be used throughout? Apart from the quantitative variations in auditory stimuli (some employed one, some two, some three, and some four different sounds), which would necessitate increasing or decreasing the number of visual shapes accordingly, there are other, more compelling reasons for varying the graphic design for each item. In one earlier study (Walker, 1978), the same set of visual alternatives was used. Subjects began to ask questions about the lack of choice; they were loath to match the same graphic design with a different configuration of the same auditory parameter. Once having used, say, the vertical metaphor for one frequency change, they felt that another graphic shape should be used for a similar parameter change that occurred at a different frequency. It was observed that, with some subjects, the use of the same graphic design throughout tended to contaminate results. In the present experiment, using a variety of graphic designs eliminated this problem, yet did not otherwise interfere with the type of visual metaphor chosen.
Something of the variety of graphic designs utilized can be seen in Figure 1. It is believed that, despite these differences in design, the actual deployment of the items (in terms of size, pattern, shape, or vertical or horizontal placement) is sufficiently clear and unambiguous to enable subjects to identify easily each class of visual deployment. The systematic choices made by the subjects, as described below, seem to justify this belief.

\section{Results and Discussion}

Many of the findings of the previous studies cited were confirmed by the results of the present experiment. Overall, the subjects displayed a proclivity for choosing systematically a visual metaphor for each of the four acoustic parameters in the following manner: frequency was matched with vertical placement, amplitude with size, waveform with pattern, and duration with horizontal length. If these matches are assumed to be the correct choices for the purposes of data analysis, the total sample scored well above guessing rates on combined total scores. On the 16-item test, with four response alternatives for each item, the $S D$ of the distribution $=\sqrt{n p q}$ $=1.732$. With $z$ scores of $1.95(p<.05)$ and 2.58 $(p<.01)$, guessing rates are calculated to be 7.39 $(p<.05)$ and $7.47(p<.01)$. All guessing rates mentioned below are calculated on the same basis. The mean of the entire sample was 11.16, which indicated that on the whole the sample could perform this test as expected. However, significant differences (MANOVA, $p<.01$ ) were observed between the musically trained $(M=14.14)$ and the other groups (combined $M=10.42$ ). Tests for differences (Scheffé) among the groups indicated that the group mean for the musically trained was significantly 


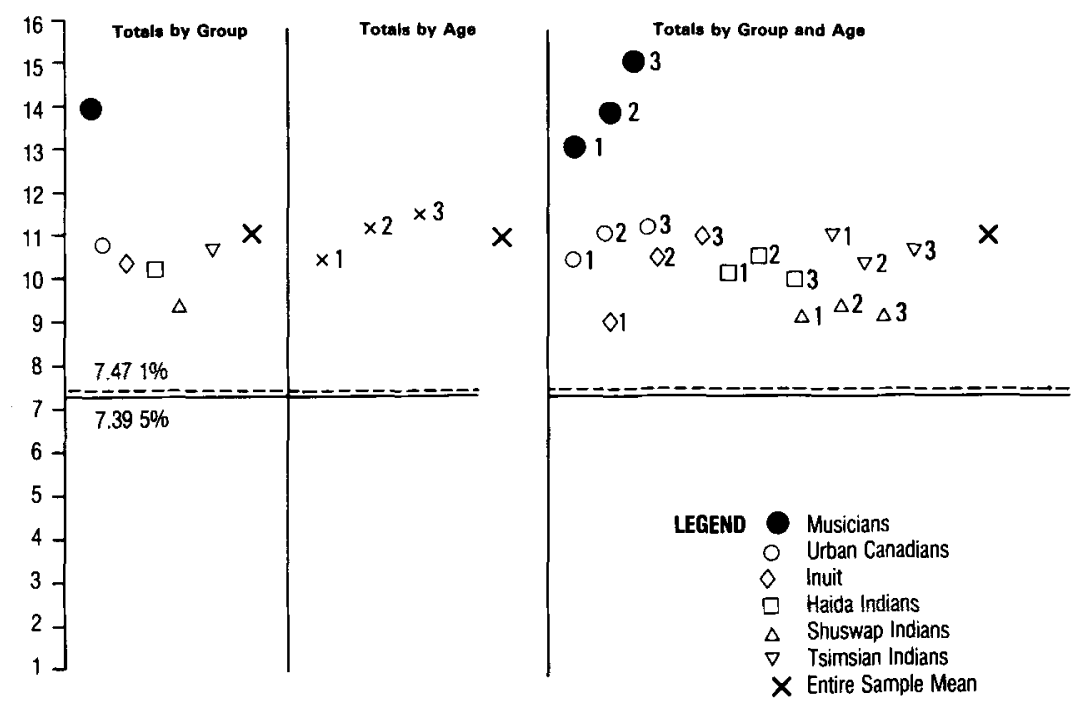

Total Group Means by Parameter

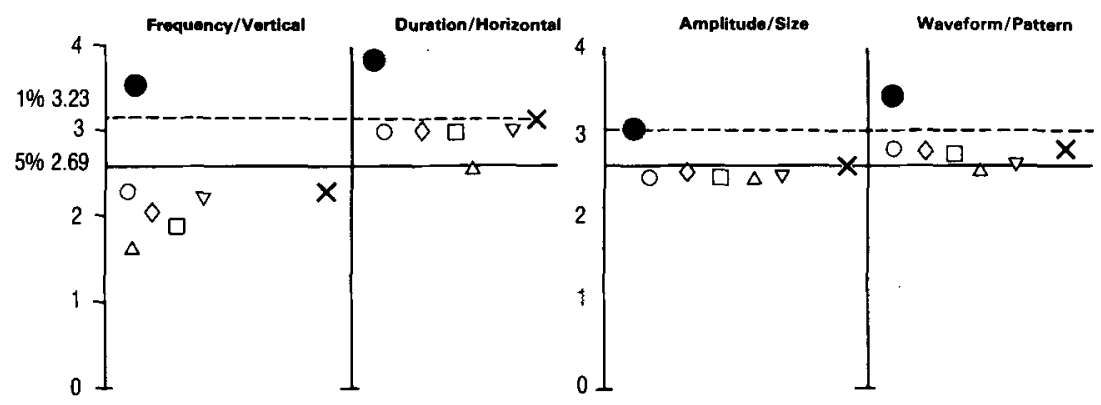

Figure 2. Total group means.

higher ( $p<.01$ in all cases) than that for the other groups, and that the group mean (9.37) for the Shuswap Indian group was significantly lower $(p<.01)$ than that for any other group. All other comparisons yielded nonsignificant $F$ ratios. This is illustrated in Figure 2 (Totals by Group). Thus, overall, there were significant effects of musical training and limited effects of culture and environment in that one Indian group made visual matches significantly different from those of other musically naive groups. This will be discussed in more detail below.

Age was seen to be a factor overall (MANOVA, $p<.01$ ), but more precise testing between groups (Scheffé) showed that most variance occurred between the total Age Levels 1 and 3 ( $p<.01$ ), with nonsignificant comparisons between Age Levels 1 and 2 and 2 and 3 . However, this occurred only within the musically trained group (Age Level 1, 2, and 3 means $=13.3,14.0$, and 15.2 , respectively) and the Inuit group $(9.22,10.86$, and 11.14). All other comparisons were nonsignificant. This is illustrated in Figure 2 (Totals by Age and Totals by Group and Age).

Significant effects of group identity were observed in comparisons (MANOVA) of all six total group means for each of the four acoustic parameters: frequency, $F(5,829)$ $=51.9, p<.001$; amplitude, $F=16.6, p<.001$; du- ration, $F=26.7, p<.001$; waveform, $F=17.3$, $p<.001$. (See Figure 2, Total Group Means by Parameter.) This can be explained mostly by the higher scores of the musically trained. Similar comparisons of total group means (MANOVA) excluding that of the musically trained group yielded nonsignificant values for $F$ for amplitude $(F=0.475)$ and waveform $(F=1.56)$, but the reverse was true for frequency $[F(4,664)=6.9$, $p<.001]$ and duration $(F=5.9, p<.001)$. Further analysis of these data (Scheffé) showed that a significantly lower mean $(p<.01)$ was scored by the Shuswap group than by three of the four musically naive groups for frequency and all four in the case of duration. No other comparisons yielded significant values. This indicates some limited effects of culture and environment. The visual response alternatives were all consistent in their use of two-dimensional space and are not considered a factor in response choices. A split-halves reliability coefficient $(r=.72)$ was obtained, indicating that the test was reliable for its intended purpose $(p<.001)$.

The sample tested comprised four basically different cultural and environmental groups. The musically trained and urban (musically naive) groups were from the same densely populated urban environment. The Haida and Tsimsian Indians share a similar coastal environment but 
in different locations, whereas the Shuswap Indians live in a completely different type of wilderness environment over 500 miles inland, have a very different language form, and are relatively isolated in their own villages from other cultural influences. The fourth group, the Inuit, live in the Arctic tundra and differ from the other groups in both environment and language culture. Thus, surprisingly perhaps, no effects of culture and environment were observed between choices made by Inuit, Tsimsian, and Haida Indians in comparisons of total group means. The Shuswap Indians displayed a greater tendency to choose visual size as a metaphor for frequency, as compared with other groups. For the total frequency items, size attracted a $39.7 \%$ response and vertical placement a $40.8 \%$ response with the Shuswap group. For the two discrete frequencies (F2 and F3; see Figure 3), this group showed a preference for size over vertical $(49.1 \%$ vs. $29.9 \%$; $\chi^{2}=10.95, p<.001$ ); for the two glides (F1 and F4), the reverse was true, with $51.7 \%$ for vertical versus $30.3 \%$ for size $\left(\chi^{2}=13.02, p<.001\right)$. The other musically naive groups displayed a slight preference for vertical over size, with significant differences occurring randomly across different groups and age levels. Differences in responses to duration stimuli can be explained by a slight inclination toward size and vertical placement among the Shuswap group. For the frequency items, all the musically naive subjects could not reach the $5 \%$ guessing rate; however, these groups selected horizontal matchings at better than guess rates for the duration stimuli. The exception was the Shuswap, whose group mean, 2.60, was marginally below the $5 \%$ guessing rate $(2.69)$.

\section{Frequency}

A more detailed examination of the results for each stimulus set provided a clearer focus on the main findings (see Figure 3). In the case of all four frequency stimulus sets, the musically trained group means (all ages combined) were significantly above those of any other group (MANOVA, $p<.001$ ). The mean for all musically naive subjects was $2.06(5 \%$ guess rate $=2.69)$, compared with 3.5 for the musically trained group. Only at Age Level 1 for Frequency Set 1 did the latter group fail to achieve significant differences above all others.

Paired comparisons (Scheffé) of the means of all five musically naive groups (combined ages) for all four frequency stimulus sets were mostly nonsignificant. These data, therefore, showed few meaningful cultural and environmental effects on response choices. Only two paired comparisons of responses to Frequency Set 2 yielded significant values: the comparisons between the Shuswap and urban groups $(p<.001)$ and the Shuswap and Tsimsian groups $(p<.05)$. Frequency Sets 1 and 4 comprised glides, or glissandi in musical terms, whereas Sets 2 and 3 comprised discrete frequencies. The higher scores for Set 4 may be a reflection of its acoustic nature. It is possible that three upward acoustic glides provide greater definition of the frequency parameter than does a single glide (Set 1), facilitating a more consistent choice of the vertical visual match. Musically naive groups scored above or near the $5 \%$ guess rate for Set 4 , whereas the musically trained group scored significantly higher (Scheffé, $p<.01$ ) in all paired comparisons. Considering evidence from a number of researchers (e.g., Rakowsky, 1971) that discrete frequency stimuli attract smaller difference limens, it might be expected that the discrete stimuli (F1 and F2) in this test would induce higher scores among the musically naive. However, discrimination of frequency change is a different kind of task from that of selecting a visual metaphor for frequency movement in general. It is clear from these data that musically naive subjects from varied backgrounds experience some difficulty in selecting a consistent visual metaphor for both discrete frequencies and some glides.

Age was a factor overall with Frequency Set 2 (MANOVA, $F=9.9, p<.001$ ) and Frequency Set 3 $(F=3.03, p=0.049)$. Age effects were also observed (Scheffé) between Levels 1 and 3 as follows: musicians in F1 and F3; Tsimsian in F1, though inverted effects between Levels 1 and 3; Shuswap in F3; and Inuit in F4.

\section{Amplitude}

Comparisons (MANOVA) of total group means for each amplitude stimulus set yielded significant values for $F$ in Amplitude Set $2[F(5,819)=19.95 ; p<.001]$ and Set $4(F=8.44, p<.001)$, but nonsignificant values for the other two sets (see Figure 3). Most of the variance in the former can be accounted for by the higher scores of the musically trained, whose sample mean was 3.19 , compared with 2.54 (below the $5 \%$ guess rate) for the entire sample of musically naive subjects. Group means were clustered above the $1 \%$ guess rate for Amplitude Set 1 and above the $5 \%$ rate for Amplitude Set 3, with no significant differences observed among any of the groups for either set. Comparisons of group means (MANOVA) for all five musically naive groups yielded nonsignificant $F$ values.

This disparity in response pattern between Amplitude Sets 1 and 3 and 2 and 4 can be explained in part by the nature of the auditory stimuli. Sets 1 and 3 comprised discrete changes in amplitude, whereas Sets 2 and 4 were continuous. It seems likely that, for the purpose of identifying the parameter of change sufficiently to make judgments concerning the suitability of visual metaphors, continuous-amplitude stimuli of this type are not as easy as discrete-change stimuli. Clearly, musical training was a factor here. In paired comparisons (Scheffé) of total group means between the musically trained group and each of the five musically naive groups, significant values for $F$ were obtained in each case $(p<.001)$. All paired comparisons between the musically naive group means were nonsignificant. These data show clearly the effects of training and lack of effects of culture and environment in respect to these amplitude stimuli. There were some effects of age for Amplitude Set 4 in the urban group and 

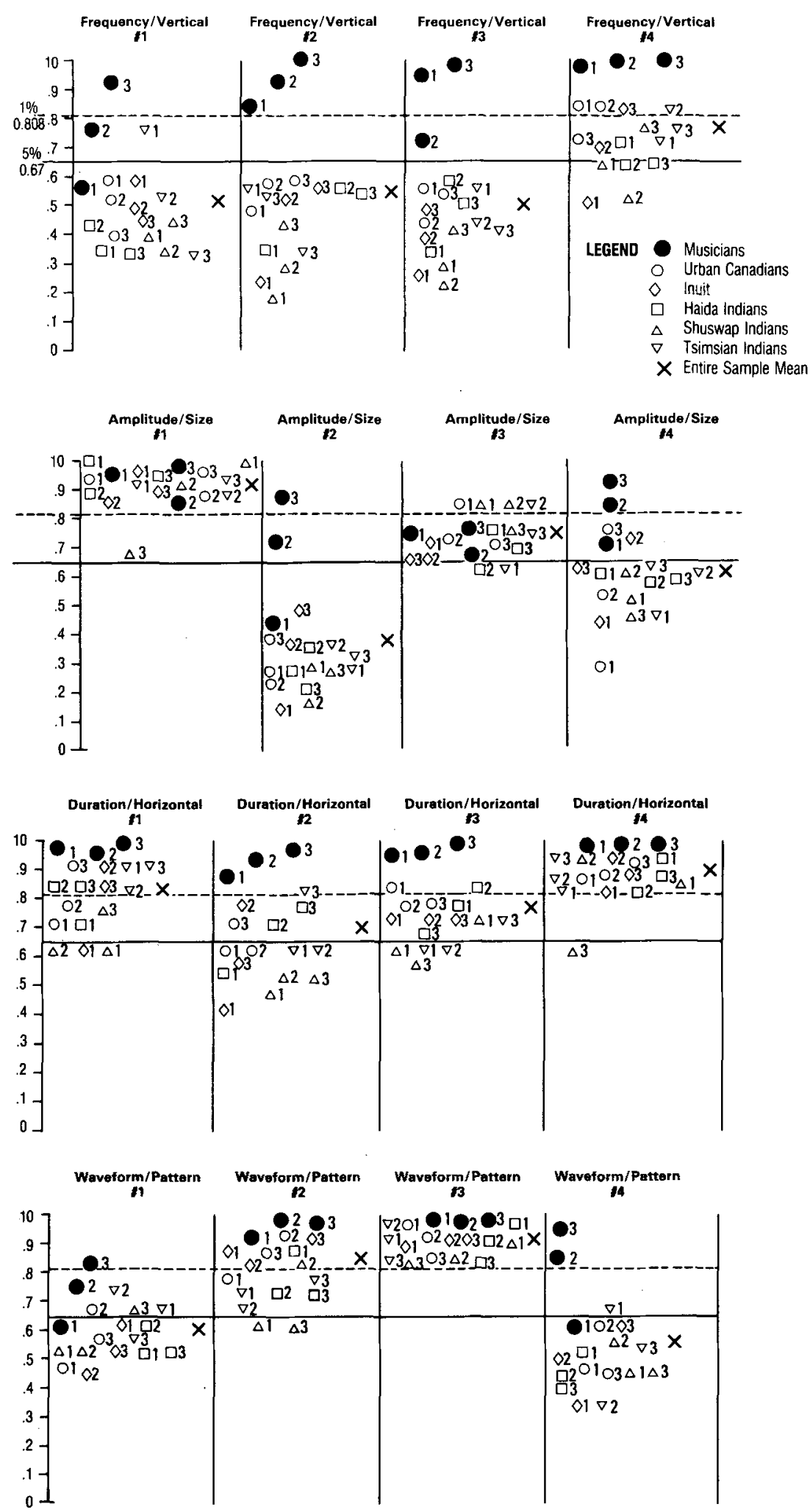

Figure 3. Age and group means for individual items. 
for Amplitude Set 2 in the musically trained and Inuit groups $(p<.01)$.

Response choices for each of the musically naive groups for Amplitude Set 2 demonstrated a preference for visual pattern over size as a metaphor $\left(\chi^{2}=32.08, p<.001\right.$ in respect to total scores). This raises the possibility that musically naive subjects heard the different Fourier components more clearly as the amplitude increased, and were unable, consequently, to fuse them perceptually, in which case perception of complexity of components was matched with pattern complexity. Age Levels 2 and 3 among the musically trained were not affected in this way. The same may be true of some responses to Amplitude Set 4, although size was preferred over pattern among the musically naive $\left(\chi^{2}=49.9, p<.001\right)$. There were no observable effects of culture and environment in analyses of group means. Therefore, it can be concluded that musical training is the most important factor in choice of visual metaphor for certain movements in amplitude.

\section{Duration}

For each duration stimulus set, the musically trained group scored significantly higher than all other groups (MANOVA; $p<.01$ ). The group mean of the former was 3.88, whereas the sample mean of the latter was 3.03. Total group means (combined ages) for each of the musically naive groups lay just below the $1 \%$ guess rate, with the exception of the Shuswap group, whose mean lay just below the 5\% level (see Figure 2). Paired comparisons of the total group means for each of the four duration sets yielded nonsignificant values for $F$, except for the comparisons between the Shuswap group and the Tsimsian group in Duration Sets 1 and 2. Overall, significant effects of group identity were observed (MANOVA, $p<.001)$ among the musically naive subjects. Further analysis (Scheffe) showed that this was due predominantly to the lower group means observed for the Shuswap group. Despite this, the Shuswap group chose the horizontal metaphor more consistently, but not more significantly, than any other. Overall, some aspects of musical training were observed, with some limited effects of culture and environment observable in respect to the Shuswap group only.

\section{Waveform}

Responses to some of these stimuli showed effects of musical training. The mean of the entire sample of musically naive subjects was 2.82 . This is higher than the $5 \%$ guess rate, but lower than the mean of the musically trained group (3.5). Effects of musical training were apparent in overall comparisons (MANOVA, $p<.001$ ) and those for each set ( $p<.001$ in each case). No effect of group identity was observed (MANOVA, $F=1.5$, $p=0.182$ ) among the five musically naive groups, overall, in results for Sets 1, 3, and 4. Some effects of group identity [MANOVA, $F(4,663)=4.26, p<.002$ ] were seen in responses to Set 2 . This can be explained by the lower Shuswap group mean (0.70) as compared with the other four: urban, 0.86; Inuit, 0.86; Haida, 0.79; Tsimsian, 0.75 .

Total group means for each musically naive group were below the $5 \%$ guess rate for Sets 1 and 4 , but above this level for the other two sets. In the latter case they were all well above the $1 \%$ guess rate. This suggests that paired sets 2 and 3 and 1 and 4 elicited a different response pattern for each pair. The nature of auditory stimuli in each case might explain these disparities. Waveform Sets 1 and 4 comprised discrete sounds that contrasted a simple with a more complex wave in each case. The visual alternatives for Set 4 can be seen in Figure 1, Number 16. Sets 2 and 3 were different in that there was greater contrast in the stimuli: a simple waveform and a complex wave combined with noise in each case. Only at Age Level 1 did the musically trained group produce means below the $5 \%$ guess rate for Waveform Sets 1 and 4; musically trained subjects at Age Levels 2 and 3 scored higher. Interestingly, the same can be said for Frequency Set 1 and Amplitude Set 2, which suggests that clearer definition of acoustic parameters in the form of clearer and greater acoustic contrast is a factor with these stimuli for musically naive subjects. Short continuous stimuli, as in Frequency Set 1, or more subtle parameter changes, as in Waveform Sets 1 and 4, tend to provide insufficient auditory information to enable musically naive subjects to select systematic visual metaphors significantly above guess rates. Despite this, the musically naive groups favored pattern change (Waveform Set $1,59.2 \%$, and Set 4 , $51.5 \%$, overall) over the nearest other choice, size (Waveform Set 1, 14.7\%, and Set 4, 34.2\%, overall). Chisquare tests of raw scores comparing the two most favored choices, accounting for around $\mathbf{8 0 \%}$ of all choices among the musically naive, yielded significant values for chisquare in all cases. This suggests that with a clearer acoustic definition in the waveform stimuli, these subjects might have chosen a visual pattern metaphor more consistently.

\section{Comments}

\section{Main Findings}

The main findings to emerge from these data are as follows: Musical training is the most important single factor in choices of visual metaphor for sounds. Clarity of delineation of acoustic parameters is a contributing factor with some stimuli to the responses of musically naive subjects, irrespective of their cultural and environmental background. Age is a factor, but of less importance. Culture and environment were found to be significant factors for one cultural group, which lived in a more isolated environment than the others. Some studies cited above showed that musical training is a significant factor where frequency is concerned; the present study extends that finding to include certain configurations of waveform, amplitude, and duration.

\section{Stimulus Modality}

There were no simple and clear-cut distinctions across the results by virtue of modality that differentiated those 
test items attracting high scores uniformly from those that did not. One modal-specific item (F4) uniformly attracted high scores, and several amodal items attracted low scores. Duration Sets 1, 3, and 4 were possible exceptions, as were Frequency Sets 1, 2, and 3. Of the four modal-specific items in the frequency category, the two discrete stimuli (F2 and F3) caused the most difficulty for all musically naive subjects. Surprisingly, the singleglide stimulus (F1) caused similar difficulty, yet the threeglide stimulus (F4) attracted high scores from all groups. Duration Stimulus Set 2 presented some problems for musically naive subjects, possibly by virtue of its greater complexity. Among the amodal items, Amplitude Stimulus Set 2 attracted some of the lowest group means in the test, as did Waveform Stimulus Set 4. Clearly, stimulus modality is not the sole predictor of difficulty in this kind of task; clarity of delineation of acoustic parameter is at least equally important.

\section{Influences of Experience and Innate Mechanisms}

Those items attracting uniformly high group means were Frequency Set 4, Amplitude Sets 1 and 3, Duration Sets 1, 3, and 4, and Waveform Sets 2 and 3. All of these presented clearer acoustic information to subjects in the form of either repeated sounds or greater acoustic contrast, both of which tended to emphasize the nature of the acoustic event. In view of the variety of backgrounds among the subjects, it can be postulated that the high group mean scores for these items are attributable to common innate neural capabilities and to some common experiential factors, possibly some basic acoustic commonalities across all types of language use. It would follow that the nature of these acoustic stimuli did not make demands outside the effects of these factors. The remaining test items-Frequency Sets 1,2, and 3, Amplitude Sets 2 and 4, Duration Set 2, and Waveform Sets 1 and 4-can be divided into two possible categories of difficulty. Difficulty of the frequency items clearly owed something to their modal-specific nature, but the acoustic events presented were of sufficient clarity. The other items listed, however, were all amodal. This would suggest that the difficulty was caused by the nature of the acoustic event. These items would share one common characteristic: insufficient clarity of information to enable musically naive subjects to categorize them appropriately. With such items, appropriate training and experience (musical) would seem to compensate for this lack of clarity, as evidenced by the higher scores for musically trained subjects. This would indicate that musical training has effects that can be seen in other auditory-visual parameters than frequency matched with vertical placement. The nature of the difficulty presented to musically naive subjects has yet to be determined. Some continuous stimuli caused as much difficulty as some discrete ones, despite a stimulus change well above known jnds.

\section{Cultural and Environmental Factors}

Statistically, there were differences between the responses of the Shuswap Indian group and the other musically naive groups tested. There are some grounds for attributing these differences to cultural and environmental factors. Ethnologically, this group of Indians is quite distinct from the Haida and Tsimsian. Historically, the Shuswap had a vastly different life-style and less of a tradition in symbolic art production such as carving, painting, and tattooing. Unlike the other groups, they traditionally lived in subterranean dwellings in winter and temporary shelters in summer. Of all the groups tested, this was the only one that was trying to recreate its historical life-style in an isolated part of its traditional lands, separated from the coast by impassable mountains and distance, and, therefore, remote from Western influences. Interestingly, a study carried out in Egypt (Sadek, 1987), using exactly the same test instrument with subjects of different levels of educational attainment and different living environments, indicated that subjects with some musical training in the Western style had group means higher than those of any other group, and, more significantly, that groups living more in the traditional Arab style in villages had lower group means than did those in urban environments. This would seem to indicate that, after a certain point of simplicity in the auditory stimuli (Amplitude Set 1 and Waveform Set 3, for example), effects of Western culture have some impact among the musically naive. The more remote from Western auditory and visual cultural artifacts the subjects, the less likely they are to select the kind of visual metaphor for sound that those trained in Western musical practices consistently select.

\section{Limitations of the Study and Suggestions \\ for Further Investigation}

The choice of auditory stimulus in the present study was motivated chiefly by a need for clear delineation of each particular acoustic parameter. Therefore, only limited variation was attempted. However, the results provided some evidence that might be useful in indicating some specific points needing further study. Previous studies had not shown with any great clarity to what purpose one might systematically vary auditory stimuli. Now, however, it is possible to suggest the following areas where more information is required.

Frequency. Three consecutive glides attracted consistent visual matching among all groups, whereas one glide (F1) did not. It is not clear why one glide played forward and backward should yield less "glide information" for visual matching purposes than would three consecutive glides. Otherwise, the data showed that these modalspecific items, particularly discrete ones, attracted better than guess rates only from subjects with musical training.

Amplitude. Discrete changes in amplitude elicited more consistent choices of visual metaphor than did continuous changes, despite the degree of change utilized in the continuous-change items. Further study of the effects of different degrees of change in continuous stimuli is needed. The data suggest that the degree of difference in energy levels for continuous items did not materially affect the nature of the task in terms of difficulty for musi- 
cally naive subjects. The evidence that complexity of waveform might be a factor needs further investigation.

Waveform. The results from discrete waveform stimuli (Waveform Sets 1 and 4) indicate that further study is required to discover more about the nature of the jnds in terms of wave complexity with such stimuli, in relation to detecting changes of spectral content sufficiently to provoke a consistent choice of pattern visual metaphor.

On a more general level, there needs to be further investigation in which auditory stimuli are not confined to the narrow range of fundamental frequencies found in human speech, as used in this study. Similarly, systematic variation of stimuli using complex tones seems to be warranted, in view of the difficulty some subjects had with frequency stimuli using pure tones. The limits of the effects of modal specificity need investigating. Furthermore, other changes to auditory stimuli could be employed, such as, for example, filtering spectral content or modulating signals. It is also possible that other types of visual metaphor might emerge in response to other types of auditory event, but this seems unlikely in view of the previous extensive studies of this phenomenon cited above.

\section{REFERENCES}

Algom, D., \& Marks, L. (1984). Individual differences in loudness processing and loudness scales. Joumal of Experimental Psychology: General, 113, 571-593.

Bryant, P. E., Jones, P., Claxton, B., Perkins, G. M. (1972). Recognition of shapes across modalities by infants. Nature, 240, 303-304.

BURns, E. M., \& WARD, W. D. (1974). Categorical perception of music intervals. Journal of the Acoustical Society of America, 55, 456.

Chung, D., \& Colavita, F. (1976). Periodicity pitch perception and its upper frequency limit in cats. Perception \& Psychophysics, 20, 433-437.

Clarkson, M. G., \& Clifton, R. K. (1985). Infant pitch perception: Evidence for responding to pitch categories and the missing fundamental. Journal of the Acoustical Society of America, 77, 1521-1528.

DE BOER, E. (1976). On the "residue"" and auditory pitch perception. In W. D. Keidel \& W. D. Neff (Eds.), Handbook of sensory physiology (pp. 479-583). Springer-Verlag.

DIVENYI, P. L. (1979). Is pitch a learned attribute of sound? Two points in support of Terhardt's pitch theory. Journal of the Acoustical Society of America, 66, 1210-1213.

Eimas, P. D., Siqueland, E. R., Jusczyk, P., \& Vigorito, J. (1971). Speech perception in infants. Science, 171, 303-306.

HALL, A. (1981). A developmental study of cognitive equivalence in the congenitally blind. Journal of Mental Imagery, 5, 61-73.

Halse, S. H., CYNX, J., \& Humpal, J. (1984). Absolute and relative pitch discrimination in serial pitch perception in birds. Journal of Experimental Psychology: General, 113, 38-54.

HARRIS, G., \& SiEGEL, J. A. (1975). Categorical perception and absolute pitch. Journal of the Acoustical Society of America, 57, 511. Houtsma, A. J. M., \& GoldsteIN, J. L. (1972). The central origin of the pitch of complex tones: Evidence from musical interval recognition. Journal of the Acoustical Society of America, 51, 520-529.

JAVEL, E. (1980). Coding of AM tones in the chinchilla auditory nerve: Implications for the pitch of complex tones. Journal of the Acoustical Society of America, 68, 133-146.

Lawson, K. R., Ruff, H. A., McCarton-Daum, C., Kurtzberg, D., \& VAUGHaN, H. G. (1984). Auditory-visual responsiveness in fullterm and pre-term infants. Developmental Psychology, 20, 120-127.

Lewkowicz, D. J., \& Turkewitz, G. (1980). Cross-modal equivalence in early infancy: Auditory-visual intensity matching. Developmental Psychology, 16, 597-607.

LEWKowICZ, D. J., \& TURKEWITZ, G. (1981). Intersensory interaction in newborns: Modification of visual preferences following exposure to sound. Child Development, 52, 827-832.

McGuIRE, I., \& TuRkEWITZ, G. (1978). Visually elicited finger movements in infants. Child Development, 49, 362-370.

Mendelson, M. J., \& Ferland, M. B. (1982). Auditory-visual transfer in four-month old infants. Child Development, 53, 1022-1027. Olsho, L. W., Schoon, C., Sakai, R., Turpin, R., \& SPERduto, V. (1982). Auditory frequency discrimination in infancy. Developmental Psychology, 18, 721-726.

Rakowsky, A. (1971). Pitch discrimination at the threshold of hearing. Proceedings of the Seventh International Symposium in Acoustics, Budapest, 3.373.

RITSMA, R. J., \& BiLSEN, F. A. (1970). Spectral regions dominant in the perception of repetition pitch. Acustica, 23, 334-339.

ROEDERER, J. G. (1979). Introduction to the physics and psychophysics of music. New York: Springer-Verlag.

Rose, S. A., GotTrried, A. W., \& Bridger, W. H. (1983). Infants' cross-modal transfer from solid objects to their graphic representations. Child Development, 54, 686-694.

RYKER, B. C. (1946). The ability to judge pitch. Journal of the Acoustical Society of America, 36, 331-346.

SADEK, A. A. M. (1987). Visualization of musical concepts. Council for Research in Music Education, Bulletin No. 91, 149-154.

Siegel, J. A., \& SiEgeL, W. (1977). Categorical perception of tonal intervals: Musicians can't tell sharp from flat. Perception \& Psychophysics, 21, 399-407.

TERHARDT, E. (1974). Pitch, consonance and harmony. Journal of the Acoustical Society of America, 55, 1061-1069.

Walker, A. R. (1978). Perception and music notation. Psychology of Music, 6, 21-46.

WALKER, A. R. (1981). The presence of internalised images of musical sounds. Council for Research in Music Education, 66-67, 107-112.

WALKER, A. R. (1985). Mental imagery and musical concepts: Some evidence from the congenitally blind. Council for Research in Music Education, 85, 229-238.

WALKER, A. R. (1987). Some differences between pitch perception by children of different cultural and musical backgrounds. Council for Research in Music Education, Bulletin No. 91, 166-170.

WalKer-ANDREWS, A. S., \& LENNON, E. M. (1985). Auditory-visual perception of changing distance by human infants. Child Development, 56, 544-548.

WARD, L. M. (1985). Mixed-modality psychophysical scaling: Interand intramodality sequential dependencies as a function of lag. Perception \& Psychophysics, 38, 512-522.

Wormtrh, S. J., Pankhurst, D., \& MoffitT, A. R. (1975). Frequency discrimination by young infants. Child Development, 46, 272-275.

(Manuscript received July 14, 1986; revision accepted for publication April 3, 1987.) 